\title{
HIGH-EFFICIENCY AUTONOMOUS LASER ADAPTIVE OPTICS
}

\author{
Christoph Baranec ${ }^{1}$, Reed Riddle $^{2}$, Nicholas M. Law ${ }^{3}$, A. N. RamapraKash ${ }^{4}$, Shriharsh Tendulkar $^{2}$, \\ Kristina Hogstrom ${ }^{2}, \mathrm{Khanh}_{\mathrm{Bui}}^{2}$, Mahesh Burse ${ }^{4}$, Pravin Chordia ${ }^{4}$, Hillol Das ${ }^{4}$, \\ Richard DeKany ${ }^{2}$, Shrinivas Kulkarni ${ }^{2}$, And Sujit Punnadi ${ }^{4}$ \\ ${ }^{1}$ Institute for Astronomy, University of Hawai ‘i at Mānoa, Hilo, HI, NZ 96720-2700, USA; baranec@ hawaii.edu \\ 2 Division of Physics, Mathematics, and Astronomy, California Institute of Technology, Pasadena, CA 91125, USA \\ ${ }^{3}$ Department of Physics and Astronomy, University of North Carolina at Chapel Hill, Chapel Hill, NC 27599-3255, USA \\ ${ }^{4}$ Inter-University Centre for Astronomy \& Astrophysics, Ganeshkhind, Pune 411007, India \\ Received 2014 April 17; accepted 2014 June 4; published 2014 July 7
}

\begin{abstract}
As new large-scale astronomical surveys greatly increase the number of objects targeted and discoveries made, the requirement for efficient follow-up observations is crucial. Adaptive optics imaging, which compensates for the image-blurring effects of Earth's turbulent atmosphere, is essential for these surveys, but the scarcity, complexity and high demand of current systems limit their availability for following up large numbers of targets. To address this need, we have engineered and implemented Robo-AO, a fully autonomous laser adaptive optics and imaging system that routinely images over 200 objects per night with an acuity 10 times sharper at visible wavelengths than typically possible from the ground. By greatly improving the angular resolution, sensitivity, and efficiency of $1-3 \mathrm{~m}$ class telescopes, we have eliminated a major obstacle in the follow-up of the discoveries from current and future large astronomical surveys.
\end{abstract}

Key words: binaries: close - instrumentation: adaptive optics - instrumentation: high angular resolution stars: statistics - techniques: image processing

Online-only material: color figure

\section{INTRODUCTION}

Extremely large astronomical surveys necessitate highly efficient follow-up instrumentation to fully characterize the large numbers of discoveries made (Kulkarni 2012). Adaptive optics imaging, which compensates for the image-blurring effects of Earth's turbulent atmosphere, is essential for these surveys: e.g., validating exoplanet candidates (Morton \& Johnson 2011; Marcy et al. 2014; Law et al. 2014), detecting and probing the properties of unresolved binary star systems (Lafrenière et al. 2007a; Metchev \& Hillenbrand 2009; Bowler et al. 2012; Terziev et al. 2013), and spatially locating and measuring supernovae in their host environments (Ofek et al. 2007; Li et al. 2011; Cao et al. 2013). Current laser adaptive optics systems offer the most flexible high-resolution-imaging capability, but their scarcity and complexity along with the high demand for observing time on large apertures limits their suitability for following up large numbers of targets (Hart 2010; Davies \& Kasper 2012). To address this need, we have engineered and implemented Robo-AO, a fully autonomous laser adaptive optics and imaging system that routinely images over 200 objects per night with an acuity 10 times sharper at visible wavelengths than typically possible from the ground. We have used Robo-AO to complete an adaptive optics survey of 715 Kepler exoplanet host candidates, revealing tentative evidence that short-period giant exoplanets are more likely than other exoplanets to be found in wide stellar binary systems (Law et al. 2014). We have also used Robo-AO observations to validate individual Kepler planet candidates (Swift et al. 2013; Muirhead et al. 2014), to investigate exotic binary systems (Law et al. 2012; Muirhead et al. 2013), and to search for companions to thousands of nearby stars.

Robo-AO systems offer the opportunity to upgrade the more than one hundred $1-3 \mathrm{~m}$ class telescopes that are currently operating without the benefits of adaptive optics. By greatly improving the angular resolution, sensitivity, and efficiency of 1-3 m class telescopes, we have eliminated a major obstacle in the follow-up of current and future large astronomical surveys. In this Letter, we describe the Robo-AO instrument (Section 2), describe the initial results from the system and its delivered image quality (Section 3), and detail the future plans for the system (Section 4).

\section{METHODS}

The Robo-AO instrument, mounted to the robotic $1.5 \mathrm{~m}$ telescope at Palomar Observatory (Cenko et al. 2006), comprises several main systems: a laser-launch system; a set of support electronics; a Cassegrain instrument package that houses a highspeed optical shutter, wavefront sensor, wavefront corrector, science instrument and calibration sources; and a single computer that controls the entire system (Baranec et al. 2013; summarized in Table 1).

The laser-launch system comprises a pulsed, $12 \mathrm{~W}, \lambda=$ $355 \mathrm{~nm}$ laser beam, coaligned with the bore-sight of the principal telescope, and focuses a seeing-limited beam waist with a $15 \mathrm{~cm}$ projection aperture to a line-of-sight distance of $10 \mathrm{~km}$. As with other ultraviolet laser systems propagating into navigable airspace (e.g., Thompson \& Teare 2002; Tokovinin et al. 2012), control measures to avoid illuminating aircraft are not required because the laser beam is unable to flash-blind pilots or produce biologically hazardous radiation levels during momentary exposures. The instrument package located at the Cassegrain telescope focus uses an opto-electrically shuttered Shack-Hartmann wavefront sensor to record the light returning to the telescope from the laser focus over a backscatter range of $450 \mathrm{~m}$. The signal-to-noise ratio of each Shack-Hartmann slope measurement ranges from 6 to 10 depending on Zenith angle and seeing conditions (6.5 electrons of detector noise in each of 
Table 1

Palomar Robo-AO Specifications

\begin{tabular}{ll}
\hline \hline Telescope & Palomar 1.5 m telescope \\
Science camera & Andor iXon DU-888 \\
EMCCD detector & E2V CCD201-20 \\
Read-noise (without EM gain) & $47.8 e^{-}$ \\
EM gain, selectable & $300,200,100,50,25$ \\
Effective read-noise & $0.16,0.24,0.48,0.96,1.9 e^{-}$ \\
Full-frame-transfer readout & 8.6 frames per second \\
Detector format & $1024^{2} 13 \mu$ m pixels \\
Field of view & $44^{\prime \prime} \times 44^{\prime \prime}$ \\
Pixel scale & 43.1 milli-arcsec per pixel \\
Observing filters & Sloan $g^{\prime}, r^{\prime}, i^{\prime}, z^{\prime}$ and $>600 \mathrm{~nm}$ \\
Tip-tilt guide star range & $2<m_{V}<16$ \\
Residual rms wavefront error & $141-218 \mathrm{~nm}$ \\
Strehl ratio at $i^{\prime}$-band & $26 \%-4 \%$ \\
LGS-AO overheads per target & $40-42 \mathrm{~s}($ typical) \\
Average telescope slew time & $40 \mathrm{~s}$ \\
\hline
\end{tabular}

four binned pixels with a signal ranging from 100 to 200 photoelectrons per subaperture). The measured tip and tilt signals are used to stabilize the laser pointing with an uplink beam-steering mirror, while the remaining atmospheric wavefront aberrations, excluding stellar image displacement, are compensated with a $12 \times 12$ actuator, $3.5 \mu \mathrm{m}$ stroke, micro-electromechanical deformable mirror. The adaptive optics control loop operates at the $1.2 \mathrm{kHz}$ frame rate of the Shack-Hartmann detector, with an effective wavefront control bandwidth of 90-100 Hz.

Robo-AO is the first astronomical adaptive optics system to operate autonomously; a master robotic sequencer controls the telescope, adaptive optics system, laser, filter wheel, and science camera; executing all operations that otherwise would have been performed manually, allowing greatly improved observing efficiency. The software to control each hardware subsystem was developed as a set of individual modules in $\mathrm{C}++$, and small standalone test programs have been created to test each of the hardware interfaces. This modular design allows the individual subsystems to be stacked together into larger modules, which can then be managed by the master robotic sequencer.

The execution of an observation starts with a query to an intelligent queue scheduling program that selects a target. The robotic sequencer will then point the telescope, while simultaneously selecting the appropriate optical filter and configuring the science camera, laser and adaptive optics system. A laser acquisition process to compensate for differential pointing between the telescope and laser projector optical axes, caused by changing gravity vectors, begins once the telescope has completed pointing at the new target. A search algorithm acquires the laser by moving the uplink steering mirror in an outward spiral pattern from center until $80 \%$ of the wavefront sensor subapertures have met a flux threshold of $75 \%$ of the typical laser return flux. Simultaneous with the laser acquisition process, the science camera is read out for $20 \mathrm{~s}$ with no adaptive optics compensation and with the deformable mirror fixed to obtain a contemporaneous estimate of the seeing conditions through the telescope. Upon completion of laser acquisition, a new wavefront sensor background image is taken, the adaptive optics correction is started and an observation with the science camera begins.

During an observation, telemetry from the adaptive optics loop is used to maintain telescope focus and detect significant drops in laser return flux. Slow drifts in the focus mode of the deformable mirror are measured and offloaded to the secondary mirror to preserve the dynamic range of the deformable mirror. Focus on the deformable mirror is measured by projecting the commanded actuator values to a model Zernike focus mode. A median of the last 30 focus values, measured at $1 \mathrm{~s}$ intervals, is calculated. If the magnitude of this value exceeds $220 \mathrm{~nm}$ peakto-valley surface of focus on the deformable mirror, equivalent to a displacement of $20 \mu \mathrm{m}$ of the Palomar $1.5 \mathrm{~m}$ telescope secondary mirror, then the secondary is commanded to change focus to null out this value. Focus corrections may not be applied more than once every $30 \mathrm{~s}$ and are restricted to less than $50 \mu \mathrm{m}$ of total secondary motion to avoid runaway focus. The laser return flux is also measured at simultaneous $1 \mathrm{~s}$ intervals. If the laser return drops below 50 photoelectrons per subaperture on the wavefront sensor for more than $10 \%$ of the values used to calculate the median focus, e.g., due to low-altitude clouds or extremely poor seeing (greater than 2 '.5), any focus correction is ignored due to the low certainty of the measurement. Additionally, if the return stays below 50 photoelectrons per subaperture for five consecutive seconds, the observation is immediately aborted, the target is marked as "attempted but not observed" in the queue, and a new target is selected for observation.

The intelligent queue is able to pick from all targets in a directory structure organized by scientific program, with observation parameters defined within Extensible Markup Language files. The queue uses an optimization routine based on scientific priority, slew time, telescope limits, prior observing attempts, and laser-satellite avoidance windows to determine the next target to observe. In coordination with US Strategic Command (USSC), we have implemented measures to avoid laser illumination of satellites. To facilitate rapid follow-up observations, we have developed new de-confliction procedures which utilize the existing USSC protocols to open the majority of the overhead sky for possible observation without requiring preplanning. By requesting predictive avoidance authorization for individual fixed azimuth and elevation ranges, as opposed to individual sidereal targets, Robo-AO has the unique capability to undertake laser observations of the majority of overhead targets at any given time.

The $1.5 \mathrm{~m}$ telescope takes, on average, $40 \mathrm{~s}$ to point to a new target, and Robo-AO requires $40-42 \mathrm{~s}$ for the laser acquisition before starting a new observation; this is a substantial improvement over manually operated laser adaptive optics systems that typically require 5-35 minutes (Amico et al. 2010; Minowa et al. 2012; Neichel et al. 2012; Wizinowich 2013). This high cadence leads to the routine nightly observation of over 200 targets.

Science observations are made with an electron multiplying CCD camera with a 44" square field of view and 0.043 pixel scale. Two six-position filter wheels are directly before the camera and are equipped with Sloan $g^{\prime}-, r^{\prime}-, i^{\prime}$-, and $z^{\prime}$-band filters (York et al. 2000) as well as a long-pass filter cutting on at $600 \mathrm{~nm}$ (LP600 hereafter), a blocking filter and room for four additional filters. The quantum efficiency of the entire system as a function of wavelength in the different filters is presented in Figure 1. A selectable electron multiplication gain factor of $25,50,100,200$, or 300 can be enabled to reduce the nonamplified read noise of $47.8 e^{-}$to as low as $0.16 e^{-}$at the cost of dynamic range. The camera is read out continually at a frame rate of $8.6 \mathrm{~Hz}$ during science observations, allowing image displacement, that cannot be measured using the laser system (Rigaut \& Gendron 1992), to be removed in software 


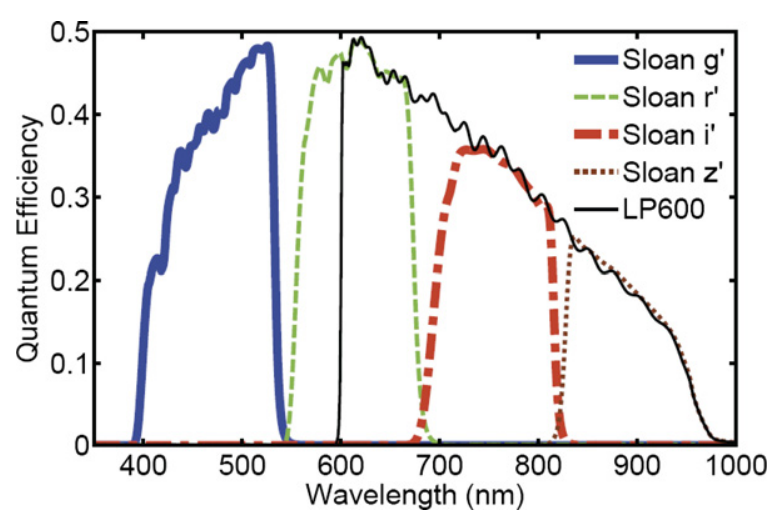

Figure 1. Quantum efficiency of Robo-AO as a function of wavelength in different observing filters. The curves are generated from measured reflection and transmission data from all optical components with the exception of the primary and secondary of the $1.5 \mathrm{~m}$ telescope which are assumed here to be ideal bare aluminum.

(A color version of this figure is available in the online journal.)

based on the position of a $m_{V} \leqslant 16$ guide star within the field of view.

Upon completion of an observation, the data is compressed and archived to a separate computer system where the data is immediately processed. A data reduction pipeline (Law et al. $2009 b, 2014)$ corrects each of the recorded frames for detector bias and flat-fielding effects, and automatically measures the location of the guide star in each frame. The region around the star is up-sampled by a factor of four using a cubic interpolation, and the resulting image is cross-correlated with a diffractionlimited point-spread function for that wavelength. This has been shown to obtain much higher quality results than centroid or brightest-pixel alignment (e.g., Law et al. 2006). The frame is then shifted to align the position of greatest correlation to that of the other frames in the observation, and the stack of frames is coadded using the Drizzle algorithm (Fruchter \& Hook 2002) to produce a final high-resolution output image sampled at twice the resolution of the input images. The rate at which final images are processed lags only slightly behind the data capture rate: a full night's set of data is typically finished before the next night of observing.

For science programs which require the detection and contrast ratio measurement of closely separated objects, an additional point-spread-function (PSF) subtraction and analysis pipeline can be started upon completion of the data reduction pipeline. This pipeline distinguishes astrophysical objects from residual atmospheric and instrumental wavefront errors and corresponding speckles in the image plane using a modified Locally Optimized Combination of Images (Lafrenière et al. 2007b) algorithm. The algorithm selects a combination of similar PSFs from the hundreds of other observations of similar targets during that night. The combination of PSFs is used to create a model PSF which is then subtracted from each image and potential companions are flagged. Figure 2 shows an example of the PSF subtraction of an automatically reduced observation that reveals a source at 0.53 away at a contrast ratio of $1 / 45$ with respect to the primary star. The PSF subtraction pipeline achieves photonnoise limited contrast of $1 / 100$ at angular distances greater than 0 .'2 from typical target stars. While this process can be fully automated, we have thus far adopted a strategy to manually verify intermediate and final results of the analysis pipeline process to ensure accurate results, e.g., initially confirming the guide star position and visually checking detected companions.

\section{RESULTS}

\subsection{Science Observations}

Robo-AO has already performed a wide range of surveys, including a comprehensive adaptive-optics survey for stellar binaries in the solar neighborhood. We have used Robo-AO to perform follow-up observations of over 3000 stars that have been identified by the Research Consortium on Nearby Stars survey (RECONS; e.g., Henry et al. 1994) to be within 25 parsecs of our Sun. Our observations will augment ongoing nearby stellar multiplicity studies by discovering stellar companions in the critical range of 1-100 AU, which are otherwise too close to be detected by seeing limited observations, or too long in orbital period to be detected by radial velocity or astrometric methods. Figure 3 shows Robo-AO observations of the first 414 of these nearby stars completed in just 2.5 nights. In this sample alone, we have discovered 37 close binary systems, and identified a possible new nearby triple star system; observations at later epochs will confirm these associations. As of this writing, Robo-AO has completed $490 \mathrm{hr}$ of fully robotic operations during 88 nights of allocated telescope time, of which $267 \mathrm{hr}$ were open-shutter science observing time. In total, the system

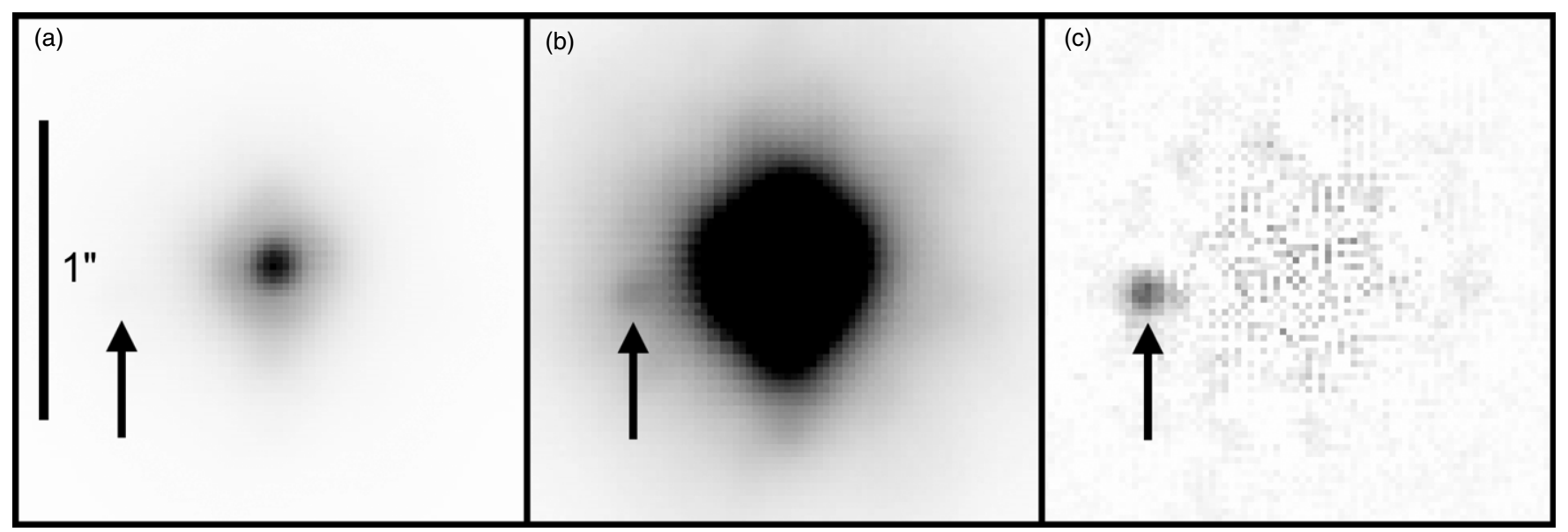

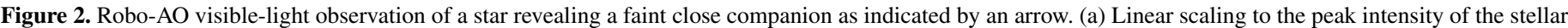

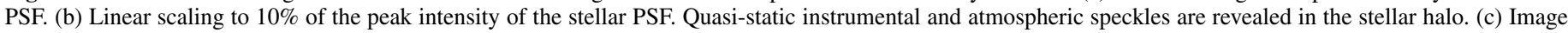
after PSF subtraction, linear scaling to $2 \%$ of the peak intensity of the primary stellar PSF. Speckles are suppressed and a faint companion is revealed. 


\begin{tabular}{|c|c|c|c|c|c|c|c|c|c|c|c|c|c|c|c|c|c|}
\hline . & , & t & , & + & , & , & , & $\theta$ & t & $*$ & * & $\boldsymbol{r}$ & * & t & , & , & , \\
\hline , & * & * & , & * & , & , & , & - & , & • & . & + & . & , & , & , & $\therefore$ \\
\hline , & , & t & , & * & , & . & , & * & $\cdot$ & , & , & , & , & , & . & 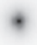 & $\cdot$ \\
\hline , & + & , & , & , & + & • & t & * & $\cdot$ & t & , & + & , & , & $t$ & 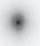 & , \\
\hline , & $t$ & , & , & , & , & , & , & , & , & $\bullet$ & , & $\cdot$ & 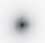 & * & , & 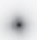 & + \\
\hline$t$ & $t$ & $r$ & , & , & 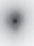 & , & $t$ & , & , & . & , & • & t & , & , & $t$ & $\cdot$ \\
\hline$t$ & , & t & t & $*$ & 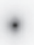 & $*$ & 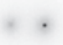 & $*$ & , & , & , & , & , & , & , & , & , \\
\hline . & . & 8 & . & , & , & a & $*$ & t & $t$ & , & , & , & , & , & , & , & , \\
\hline . & . & . & , & $*$ & $\theta$ & , & . & , & , & , & t & $*$ & $*$ & $t$ & * & $v$ & * \\
\hline . & . & $v$ & $\cdot$ & $*$ & * & • & 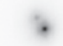 & . & $\cdot$ & , & v & , & $t$ & , & , & $t$ & , \\
\hline , & , & $*$ & $\dot{*}$ & , & $*$ & 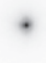 & * & • & • & , & , & , & . & , & . & , & . \\
\hline+ & . & - & , & t & + & + & t & , & t & . & t & • & , & , & , & t & , \\
\hline , & + & , & 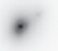 & + & , & $*$ & , & * & $\cdot$ & 5 & t & + & * & t & $t^{4}$ & , & , \\
\hline , & * & $*$ & , & * & * & a & $t$ & t & 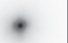 & + & • & + & * & • & $\cdot$ & + & + \\
\hline , & * & * & , & , & , & , & , & , & , & , & , & + & $*$ & , & • & • & • \\
\hline , & ${ }^{\circ}$ & + & , & • & , & , & . & , & , & + & , & * & , & $*$ & t & + & $*^{*}$ \\
\hline$t$ & , & . & t & t & . & * & , & , & , & , & + & * & + & + & + & + & $*$ \\
\hline , & , & , & , & , & , & , & , & , & 4 & . & . & • & , & , & , & . & , \\
\hline , & , & $*$ & , & $v$ & , & , & , & t & , & , & 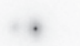 & i & . & . & , & , & . \\
\hline * & * & + & + & * & t & , & $r$ & t & , & $t$ & . & . & . & , & , & . & . \\
\hline * & ${ }^{*}$ & t & * & * & $*$ & * & , & , & + & , & • & , & , & , & * & t & , \\
\hline , & , & r & t & , & , & , & , & , & , & , & + & , & * & , & , & , & , \\
\hline t & , & , & , & $f$ & , & , & , & v & , & , & , & , & , & , & , & . & , \\
\hline
\end{tabular}

Figure 3. Robo-AO adaptive optics images of 414 stars within 25 parsecs of the Sun. Each square represents a $3^{\prime \prime} \times 3^{\prime \prime}$ area and $90 \mathrm{~s}$ of integration in the $i^{\prime}$ band, $\bar{\lambda}=765 \mathrm{~nm}$. 

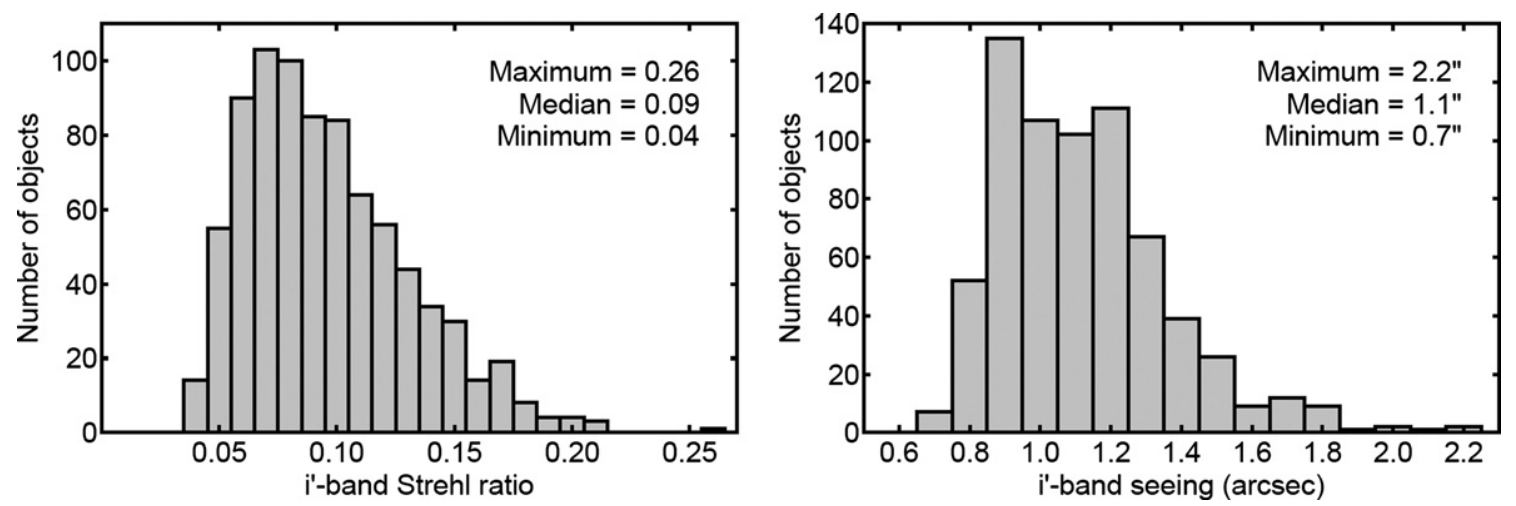

Figure 4. Summary of adaptive optics corrected $i^{\prime}$-band Strehl ratios (left) and contemporaneous seeing measurements (right).

has completed approximately 10,000 science observations, with typical exposure times ranging from 30 s to 3 minutes each.

\subsection{Image Quality}

The sharpest achieved image widths over the course of initial commissioning were measured to be $0.25,0^{\prime \prime} .10,0.12$, and $0^{\prime} .14$ in the Sloan $g^{\prime}-, r^{\prime}-, i^{\prime}$-, and $z^{\prime}$-bands respectively; in $r^{\prime}$, $i^{\prime}$, and $z^{\prime}$ these resolutions correspond to the diffraction limit of the $1.5 \mathrm{~m}$ telescope. The long-term $i^{\prime}$-band image quality improvement of the adaptive optics correction was measured as part of our program to search for stellar binaries in the solar neighborhood and is summarized in Figure 4. The 812 adaptive optics corrected observations reported here, each 90 or $120 \mathrm{~s}$ in total exposure time, are drawn from a total of 61 different nights, spanning 2012 June 17 to 2013 October 23 . They exclude observations from other science programs, identified multiple stars, objects too faint for the automatic image registration algorithm (see Law et al. 2012 for an example of observations of stars $m>16$ ), or objects that are too bright, typically $m_{V}<2$. For comparison to observing conditions, seeing limited data were taken during the time of laser acquisition prior to each of the adaptive optics corrected observations after 2012 July, comprising 682 contemporaneous seeing measurements. While our median $i^{\prime}$-band seeing value of $1^{\prime \prime} .1$ may underestimate the true seeing due to the short $20 \mathrm{~s}$ exposure times used, it is comparable to the average $\sim 1^{\prime \prime}$. $1 R$-band seeing found by Cenko et al. (2006). Under the measured observing conditions, we find the residual root-mean-square optical wavefront errors to be in the range of 141-218 nm, with a median of $189 \mathrm{~nm}$, equivalent to $i^{\prime}$-band Strehl ratios between $26 \%$ and $4 \%$, with a median of $9 \%$. Significant image sharpening is achieved under the vast majority of conditions, and only as the seeing exceeds 1". 6 does the delivered image quality fall below $5 \%$ Strehl.

\section{FUTURE PLANS}

New low-noise infrared cameras will soon be tested with the Palomar Robo-AO instrument which, once deployed, will enhance the effective near-infrared sensitivity of a $1.5 \mathrm{~m}$ telescope to that of a seeing limited $4 \mathrm{~m}$ telescope, and enable deeper visible-light imaging using adaptive-optics sharpened infrared tip-tilt guide sources. Additional instrumentation, e.g., an integral field spectrograph to quickly classify supernovae in crowded fields, can also be mounted to external instrument ports to take advantage of the automated adaptive optics correction.

Future Robo-AO systems on dedicated telescopes will be able to complete tens of thousands of high acuity science observations in a single year, making statistically meaningful discover- ies during the follow-up characterization of large surveys, and will be able to respond immediately to observation requests from transient discovery machines. We have begun work on two new facility Robo-AO systems, which we expect to be operational in 2016-2017, one for the IUCAA Girawali Observatory $2 \mathrm{~m}$ telescope in Maharashtra, India, and another for the University of Hawai'i 2.2-m telescope on Maunakea in Hawai'i. We intend to use the new systems to image transiting exoplanet host candidates discovered by the Kepler $\mathrm{K} 2$ mission (Howell et al. 2014) and the Transiting Exoplanet Satellite Survey (Ricker et al. 2014), along with candidates produced by ground-based surveys (e.g., Law et al. 2013). By identifying and measuring the components contributing to the photometric light curves, we will be able to refine the transit properties and validate thousands of potential exoplanet candidates en masse. We will also develop an interruptible queue and partner with transient surveys, e.g., the Asteroid Terrestrial-impact Last Alert System (Tonry 2011) and the Palomar Transient Factory (Law et al. 2009a), to minimize the time between discovery and characterization of rapidly changing transient events.

We thank the anonymous referee for their particularly useful suggestions. We thank the staff of Palomar Observatory for their support in the deployment of the Robo-AO system on the $1.5 \mathrm{~m}$ telescope. The Robo-AO system is supported by collaborating partner institutions, the California Institute of Technology and the Inter-University Centre for Astronomy and Astrophysics, by the National Science Foundation under grant Nos. AST-0906060, AST-0960343, and AST-1207891, by a grant from the Mt. Cuba Astronomical Foundation and by a gift from Samuel Oschin. C.B. acknowledges support from the Alfred P. Sloan Foundation. C.B., R.R., and N.M.L. wrote the paper. C.B. led the project. R.R. led the robotic software development. N.M.L. acted as project scientist and developed the data reduction pipeline. All authors contributed to the development of the Robo-AO instrument.

Facility: PO:1.5m (Robo-AO)

\section{REFERENCES}

Amico, P., Campbell, R. D., \& Christou, J. C. 2010, Proc. SPIE, 7737, 77370A Baranec, C., Riddle, R., Law, N. M., et al. 2013, J. Vis. Exp., 72, e50021 Bowler, B., Liu, M., Shkolnik, E. L., et al. 2012, ApJ, 753, 142 Cao, Y., Kasliwal, M. M., Arcavi, I., et al. 2013, ApJL, 775, L7 Cenko, S. B., Fox, D. B., Moon, D.-S., et al. 2006, PASP, 118, 1396 Davies, R., \& Kasper, M. 2012, ARA\&A, 50, 305 Fruchter, A. S., \& Hook, R. N. 2002, PASP, 114, 144 Hart, M. 2010, Appl. Opt., 49, D17

Henry, T. J., Kirkpatric, J. D., \& Simons, D. A. 1994, AJ, 108, 1437 
Howell, S. B., Sobeck, C., Haas, M., et al. 2014, PASP, 126, 398

Kulkarni, S. R. 2012, in IAU Symp. 285, New Horizons in Time-Domain Astronomy, ed. R. E. Griffin, R. Hanisch, \& R. Seaman (Cambridge: Cambridge Univ. Press), 55

Lafrenière, D., Doyon, R., Marois, C., et al. 2007a, ApJ, 670, 1367

Lafrenière, D., Marois, C., Doyon, R., Nadeau, D., \& Artigau, E. 2007b, ApJ, 660,770

Law, N. M., Carlberg, R., Salbi, P., et al. 2013, AJ, 145, 58

Law, N. M., Hodgkin, S. T., \& Mackay, C. D. 2006, MNRAS, 368, 1917

Law, N. M., Kraus, A. L., Street, R., et al. 2012, ApJ, 757, 133

Law, N. M., Kulkarni, S. R., Dekany, R. G., et al. 2009a, PASP, 121, 1395

Law, N. M., Mackay, C. D., Dekany, R. G., et al. 2009b, ApJ, 692, 924

Law, N. M., Morton, T., Baranec, C., et al. 2014, ApJ, in press (arXiv:1312.4958)

Li, W., Bloom, J. S., Podsiadlowski, P., et al. 2011, Natur, 480, 348

Marcy, G. W., Isaacson, H., Howard, A. W., et al. 2014, ApJS, 210, 20

Metchev, S., \& Hillenbrand, L. 2009, ApJS, 181, 62

Minowa, Y., Hayano, Y., Terada, H., et al. 2012, Proc. SPIE, 8447, 84471F
Morton, T. D., \& Johnson, J. A. 2011, ApJ, 738, 170

Muirhead, P. S., Becker, J., Feiden, G.A., et al. 2014, ApJS, in press (arXiv:1406.2718)

Muirhead, P. S., Vanderburg, A., Shporer, A., et al. 2013, ApJ, 767, 111

Neichel, B., Rigaut, F., Serio, A., et al. 2012, Proc. SPIE, 8447, 84474Q

Ofek, E. O., Cameron, P. B., Kasliwal, M. M., et al. 2007, ApJL, 659, L13

Ricker, G. R., Winn, J. N., Vanderspek, R., et al. 2014, Proc. SPIE, 9143, 9143508

Rigaut, F., \& Gendron, G. 1992, A\&A, 261, 677

Swift, J. J., Johnson, J. A., Morton, T. D., et al. 2013, ApJ, 764, 105

Terziev, E., Law, N. M., Arcavi, I., et al. 2013, ApJS, 206, 18

Thompson, L. A., \& Teare, S. W. 2002, PASP, 114, 1029

Tokovinin, A., Tighe, R., Schurter, P., et al. 2012, Proc. SPIE, 8447, 84474H

Tonry, J. L. 2011, PASP, 123, 58

Wizinowich, P. 2013, PASP, 125, 798

York, D. G., Adelman, J., Anderson, J. E., Jr., et al. 2000, AJ, 120, 1579 